\title{
Design and Assembly of APFC Electrical Panel
}

\author{
${ }^{1}$ Manthan , ${ }^{2}$ Bhavy,${ }^{3}$ Devang,${ }^{4}$ Rutvik \\ ${ }_{1,2,3,4}$ B.tech Student ,
}

Department of Electrical engineering, Indus university, Ahmedabad, Gujarat, India

\begin{abstract}
As we know that power system load is increasing day by day as the industrial and commercial load are increasing . In modern power system most of the load is inductive load to compensate this inductive load capacitive load is must require . Inductive load is consume reactive power in the system which affect generation of plant (in power system load is dynamic as it change according time) . the main application of apfe panel is it has automatic capacitor which connected according to requirement of reactive power.
\end{abstract}

Key word-Power Factor, APFC Panel, Power factor correction, Inductive, Capacitor, penalty

\section{INTRODUCTION:}

The power factor is the ratio between $\mathrm{KW}$ and KVA generated by the electric load, where KWis the active power and KVA is the apparent power. This is a measure of how current is effectively converted to work output and is a good indicator of the impact of load current, especially on operating system supply. Most art burdens are a stimulus to the environment that results. Disconnection of electrical components results in power outages and waste resulting in high power bills and heavy fines from the power board. When the weight of the inequality is very high, it is very difficult to maintain the unity element. To overcome this problem, APFC panels were used, retaining elements of unity. As such, industries require automatic electronic correction systems . APFC or automatic factor control panel panels are widely used for power factor optimization. Power factor can be defined as the ratio of apparent energy to active energy, and an important factor in measuring power consumption is the fact that everyone knows how much electricity is available today. Thus, in order to reduce costs, it is important to reduce the cost of electricity. To achieve this end, APFC panels are actually easily accessible. The use of these control panels is important in industries where power installations are used to supply a wide range of electronics. Decreased power factor may attract those responsible for power supply, operating loss and fines from power supply. APFC panels can effectively and automatically control rapid change and load loads while maintaining a high power factor. It will use existing ratings to respond to various requests.

\section{ii. DETAILS OF APFC}

\subsection{Power factor without using APFC Panel}

\author{
Prof. Kaushal Patel \\ Assistant Professor: \\ Dept. of Electrical Engineering \\ Indus University \\ Ahmedabad, Gujarat, India
}

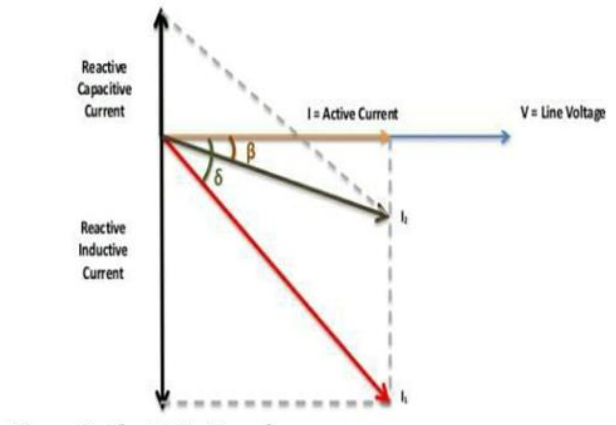

Figure.1. Electricity Board

Most industrial loads have a character, ie inductive loads, so the power factor is low or very poor depending on the power of the reactive power. These reactive loads include electric motors, induction heaters, furnaces, lamp ballasts, and the like. These loads cause the currents to hang at certain angles, leading to a poor power factor. This low power factor attracts high internal current, which in turn creates excess heat in the equipment, a huge voltage drop and poor voltage regulation. A low power factor requires a high KVA for the equipment, so the cost of the equipment increases. Therefore, energy waste will increase. There for the electricity board put extra penalty on those industries. Keeping the above things in mind, one can say that the power factor must be corrected for a better utilization of the power, and to overcome all the drawbacks discussed above there for the APFC panel will comes in to play to improve the power factor and reduces the penalty from the electricity board.

\section{iii. METHODS OF IMPROVING POWER FACTOR \\ 1 phase advanser \\ 2.synchronous condenser \\ 3. using capacitor bank \\ 1.PHASE ADVANSER:}

This method helps improve the power factor is also known as a phase advisor using an $\mathrm{AC}$ exciter. However, it can only be used for induction motors because the stator of the motor draws the winding current that is $90^{\circ}$ behind the voltage and results in a lower power factor. The only way to get rid of this problem would be to use an external source that would provide exciting ampere-winding. The phase advisor helps solve this problem when it is connected to the motor's rotor circuit. The exciting ampere-turns provided by the consultant are at slip frequency. A leading 
power factor can also be obtained by providing a greater ampere-turn.

The main advantages of using a phase advisor include the low amount of reactive power drawn by the motor. It can also be used in places where a synchronous condenser is unacceptable. However, phase motors cannot be used for motors below $200 \mathrm{hp}$. Which is informal.

2.SYNCHRONOUS CONDENSER : Another way to improve the power factor is to use a 3-phase synchronous motor, which is over-excited and operates without load. This setup is known as a synchronous condenser. The interesting part is that synchronous motors can operate under pioneer, lagging, or unity power factor. If an inductive load is present, the condenser will be connected to the edge of the load and act as a capacitor to correct the power factor. The synchronous condenser has many advantages as it requires less maintenance, can last up to 25 years and is not affected by harmonics. However, its disadvantages include high maintenance, cost and noise. Additional equipment is also required to start the motor as it does not have self-starting torque.

\section{USING CAPACITOR BANK -}

A well-known factor that decreases the power factor is the current due to the inductive load. To improve the power factor, static capacitors are installed parallel to devices operating at low power factor. The major current drawn by such capacitors neutralizes or corrects the lagging reactive component of the load current. Static capacitors have many advantages because they are lighter, easier to install, have less damage and require less maintenance. However, the losses are quite notable where if the voltage is exceeded the capacitors will be damaged quickly and their repair will become expensive. He also has a short service life (8-10 years).

\section{iv.BASIC THEORY OF APFC PANEL}

\subsection{Needs for automatic power correction}

Power factor correction (PFC) is a technique of counteracting the undesirable effects of electric loads that create a powerfactor that is less than one.Power factor correction may be applied either by an electrical power transmission utility to improve the stability andefficiency of the transmission network or correction may be installed by individual electrical customers to reduce the costscharged to them by their electricity supplier.An electrical load that operates on alternating current requires apparent power, which consists of real power plusreactive power. Real power is the power actually consumed by the load. Reactive power is repeatedly demanded by the loadand returned to the power source, and it is the cyclical effect that occurs when alternating current passes through a load thatcontains a reactive component.Power factor correction attempts to adjust the power factor of an AC load or an AC power transmission system tounity through various methods. Simple methods include switching in or out banks of capacitors or inductors which act tocancel the inductive or capacitive effects of the load, respectively. For example, the inductive effect of motor loads may beoffset by locally connected capacitors.
v. DESIGN METHODOLOGY
Panel design comprising following steps
1. GA drawing
2. meter wiring
3. control wiring diagram .
4. Single line diagram

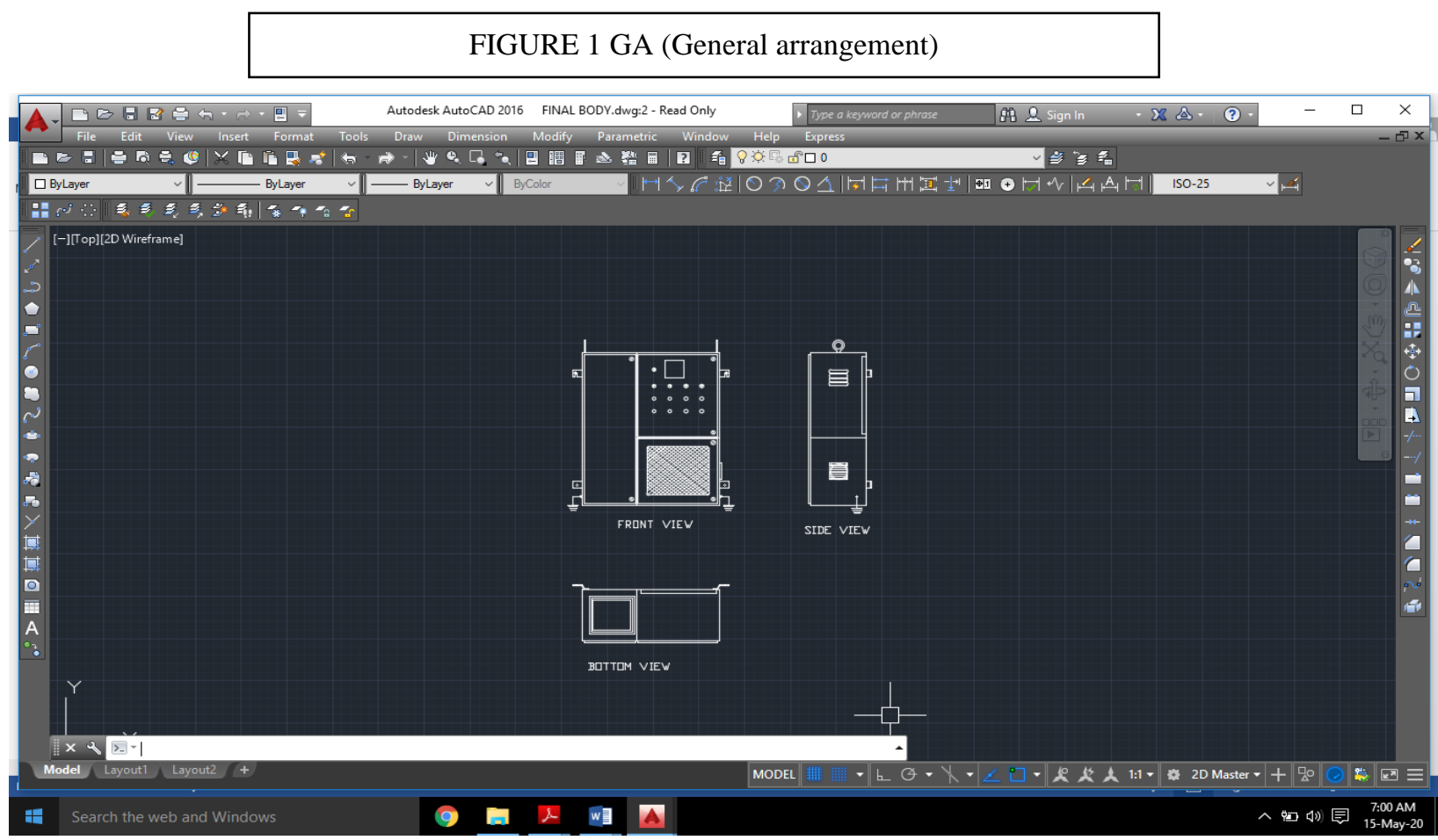


FIGURE 2 (METER WIRING) wwwWIRING)
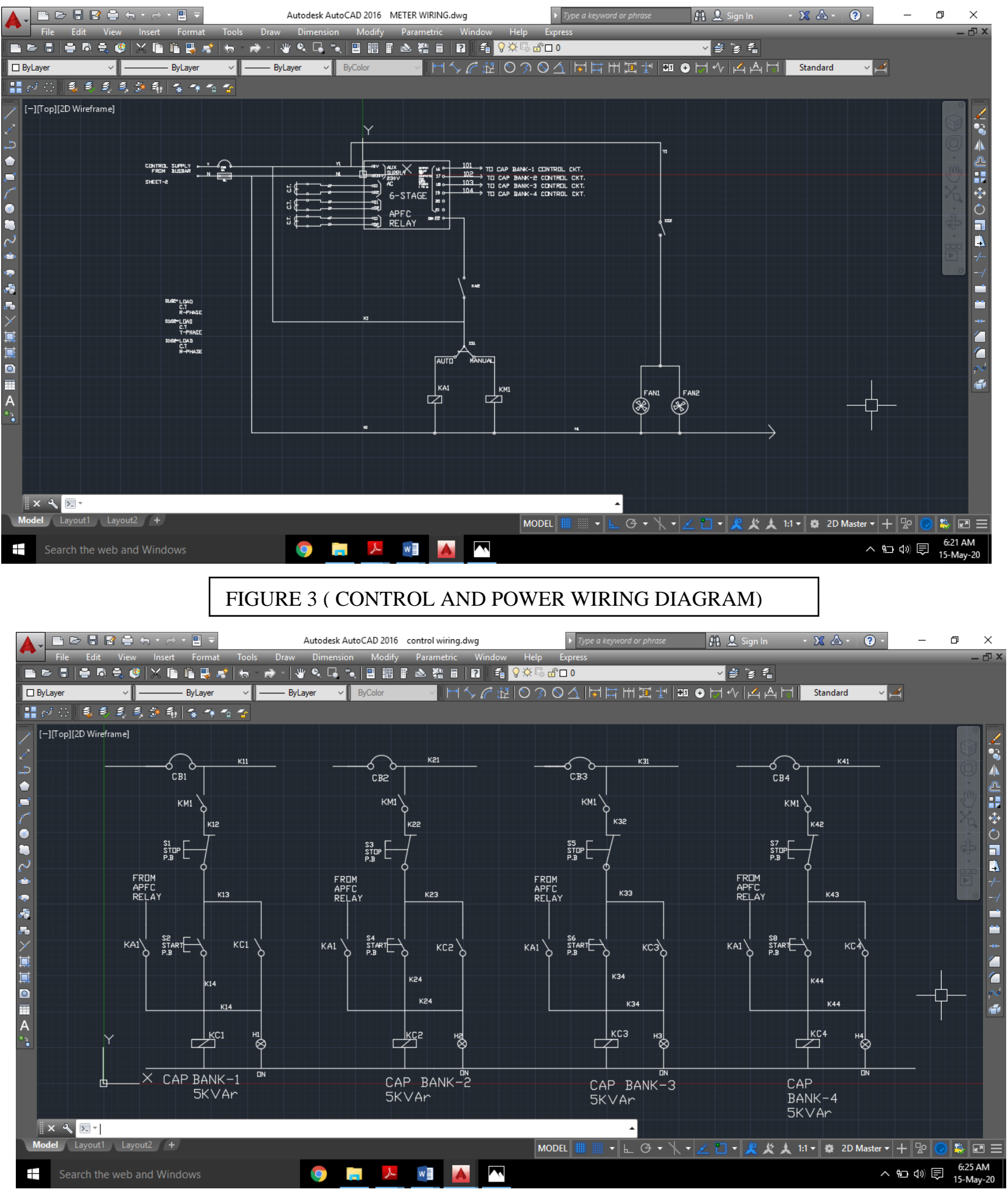


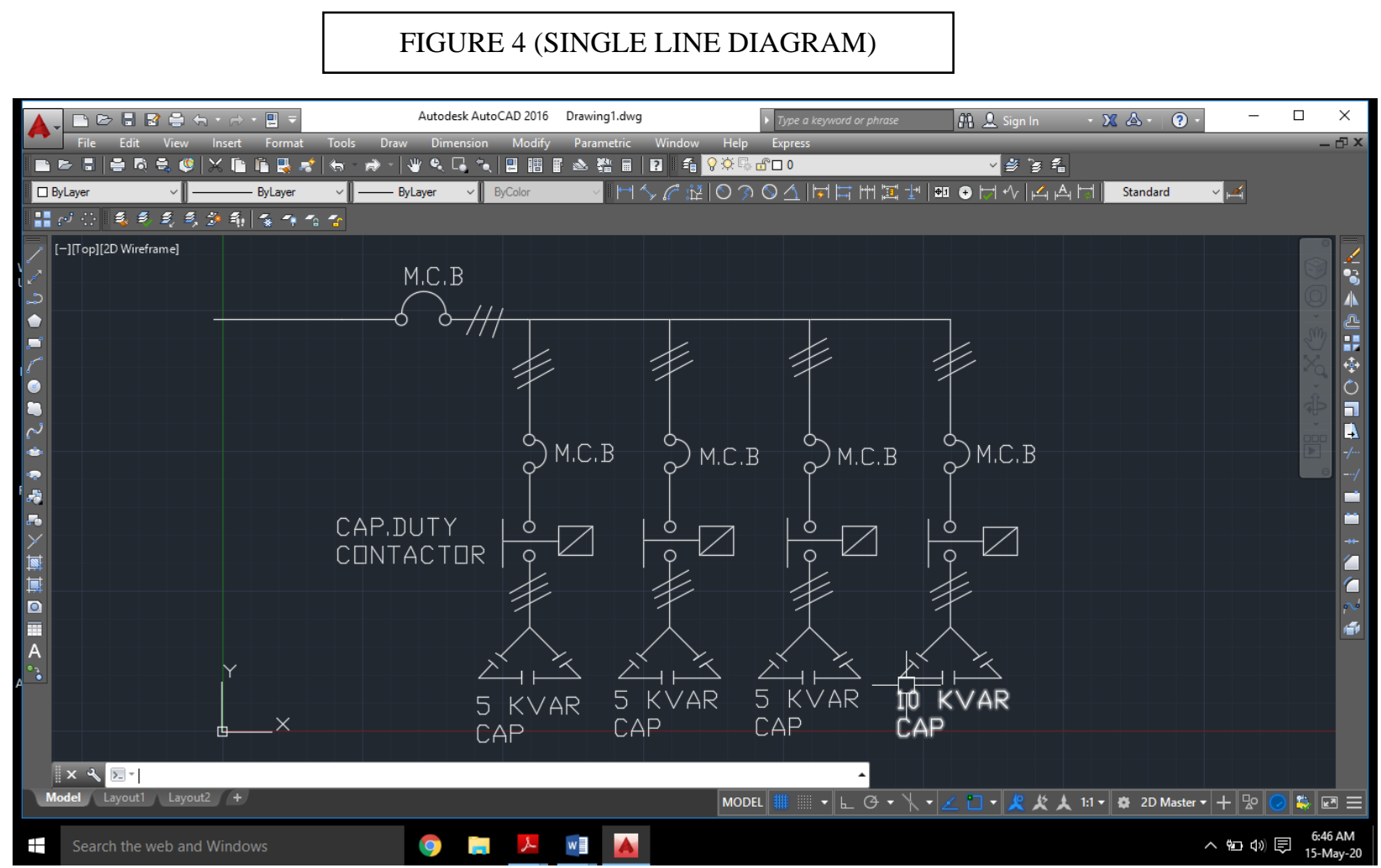

- WORKING

Meter and controlled wiring diagram Figure no. (2) shows the meter wiring and figure (3) shows the controlled wiring . Three c.t. connected with three phase and measure current through and as per reactive power requirement relay gives the output signal to no duty contactor as per requirement the duty contactor on or off simultaneously. In relay auxiliary power supply is phase to phase .one phase is going to auto manual switch and second phase going to cooling fan .In the auto manual switch to contactor is connected with 2 switch point. First point ka1 and second point KM 1when auto manual switch terminal is autoside then KA1

contactor is energize figure (3) shows the ka1 is off when contactor energize ka1 is converted in $\mathrm{NC}$ (normally closed ) in all four bank and as per reactive power requirement apfc relay pass the output signal to any kc contactor and kc contactor energize than capacitor duty contactor is convert $\mathrm{NO}$ (normally open ) to NC contactor and delta connected capacitor is fed the reactive power in supply line .

AS in figure -4 shows that three 5 kva capacitors and one 10 kvar capacitor 4 bank apfc panel designed in the system require 15 kvar supply when apfc relay give signal to duty contactor there after $\mathrm{kcl}$ contactor will energize as per figure (3) in $\mathrm{kc1}$ contactor connect with $5 \mathrm{kvar}$ delta connected capacitor so 5 kvar fed in the supply line but require 15 kvar the $\mathrm{kc} 2$ contactor is energize and another capacitor is connected means $15 \mathrm{kvar}$ demand is not connect with supply till then apfc relay gives the signal to duty contactor.
- Manual connection

When auto manual switch manual terminal side $\mathrm{km} 1$ is energize and convert in no to nc then as per figure (3) $\mathrm{km} 1$ works as nc when we press the start button PB (push button) then $\mathrm{kc}$ contactor is energized and $\mathrm{KC}$ contactor work NO to $\mathrm{NC}$ and capacitor connect with the supply this process is totally is manually and to disconnect the capacitor press the stop P.B then $\mathrm{KC}$ contactor dienergize then capacitor will disconnect.

This manual system is use for continuous flow of reactive power.

\section{vi.CONCULSION}

By installing suitable capacitor bank into circuit the power factor is improved the value become nearer to 0.9 to 0.95 consequently, it minimize loss of power system and improve efficiency of system . by using the APFC panel system become more stable and reliable also, efficiency of power system is highly increased . capacitor banks have generated reactive power and requirement of reactive power drawl from system therefore consumers can better utilize the power supply by using apfc panel and optimize use of power in power system .

\section{vii. REFERENCE}

[1] http://www.electricalnotes-article.com by "Jignesh Parmar

[2] power electricals

[3] http://www.electricalnotes-apfcblockdiagram.com/

[4] International standards IEC 60831-1

[5] International standards IEC 60831-2

[6] J. Dungan Glover, Mulukts S.Sarma, Thomas G. Overbye."Power System Analysis and Design",

[7] [POWER FACTOR CORRECTION .] www.nhp.com.au. NHP catalogue -PFC

[8] http://www.electrosome.com 